\title{
Resolving the equation between mucormycosis and COVID-19 disease
}

\author{
Ritu Pasrija ${ }^{1}$ (D) Mohammad Naime ${ }^{2}$
}

Received: 17 September 2021 / Accepted: 10 December 2021 / Published online: 22 January 2022

(c) The Author(s), under exclusive licence to Springer Nature B.V. 2021

\begin{abstract}
The COVID-19 patients, both infected and recovered are rapidly contracting mucormycetes infections due to the 'Mucorales' order, under Zygomycetes class of fungi. The mucorales fungi commonly known to exist in our natural surroundings including soil, but the frequency of incidences was never rampant. This sudden spike in infections, is locally known as 'black fungus,' and is affecting various organs, including- eyes, sinuses, nose, brain, skin, intestine, lungs, etc. The severity of situation is ascertainable from the fact that, in certain cases surgical eye/jaws removal persists as the only viable option to avert mortality, as therapeutic interventions are limited. This epidemic situation intrigued experts to investigate the probable reason behind this unpredicted escalation in reported cases, including in recuperated COVID-19 patients, as person-toperson spread of infection is not common. The comparison of physiological parameters in healthy and COVID-19 afflicted patients highlights that the underlying conditions including diabetes mellitus, steroidal therapy, lymphopenia (decreased CD4+ and CD8+lymphocytes), deregulated cytokine release storm, elevated free iron levels (hemosiderosis) in blood and insulin insensitivity are playing major roles in deteriorating conditions in rarely pathogenic fungal infections. This review is an attempt to explain the rationalities that makes people vulnerable to mucormycetes infection.
\end{abstract}

Keywords Mucor · COVID-19 · Black fungus · IL-6 · Ferroptosis · Angioinvasion · Lymphopenia · SOCS-3 · JAK-STAT · Diabetes mellitus $\cdot$ IRS-1 $\cdot$ Akt signalling $\cdot$ GLUT-4

\section{Introduction}

The COVID-19 patients, either recovering or now salvaged, are contracting fungal infections in post complications. These infections are instigated by a wider fungal group, called mucormycetes, and disease is termed as COVID19 associated mucormycosis (CAM) [1]. Although, it is reported as 'Black fungus' in news statements, due to its dark pigmentation, but is scientifically incorrect, as 'dematiaceous' fungi, are rather called as black fungi, which are far less fatal than mucormycetes [2]. The mucormycetes infections are leading to eye sight loss, jaw and teeth infections, often followed by need of subsequent surgeries to remove

Ritu Pasrija

ritupasrija@yahoo.com;

ritupasrija.biochem@mdurohtak.ac.in

1 Department of Biochemistry, Maharshi Dayanand University, Rohtak, Haryana, India

2 Central Research Institute of Unani Medicine (Under Central Council for Research in Unani Medicine, Ministry of Ayush, Govt. of India), Lucknow, Uttar Pradesh, India eyes/jaws, and death in many cases [3]. The mucormycosis related mortality is $35-50 \%$ in afflicted people [1]. In a year, almost 45,000 cases of mucormycosis are already reported [4]. Most of the cases have been reported from India, which coincidently has second highest number of diabetic people in the world [5]. Besides that, France, Iran, Russia, Pakistan, Bangladesh, Mexico, Oman, Egypt, Argentina, United States of America etc. have reported cases of this fungus as well $[4,6-10]$.

Conflictingly, mucormycetes infections are usually rare, as these are saprophytic, ubiquitous fungi and regularly found in soil, air, manure, wood and decaying matter [8]. This fungus is sometimes found in the flora of nostrils and mucus of healthy people (immunocompetent), but doesn't result in illness [9]. Thus, it was startling to observe the acute rise of these fungal infections in COVID-19 patients. Additionally, like SARS-CoV-2 infection, males were found to be more inflicted with mucormycetes infections than females counterparts $[10,11]$. Thus, it became imperative to understand the changes in physiology of pandemic struck patients, which results in an acute rise in incidences and mortality in COVID-19 patients, even after they have recovered. In 
this review, we have attempted to understand the fungus, its classification, symptoms, life cycle, mechanism of infection and lastly the factors that support the violent increase in its spread among SARS-CoV-2 infected patients.

\section{Classification and symptoms}

Mucormycetes are non-septate filamentous fungi (mold) and its colonies are cottony white or greyish black in colour. The mucormycosis (previously known as Zygomycosis (for zygospore formation)), first explained in 1855 , is caused by Mucorales order, which is distributed into eleven genera and 27 species [7]. Infections from any of these species come under mucormycosis, but Rhizopus spp, including$R$. arrhizus, $R$. homothallicus, $R$. oryzae, $R$. pusillus and $R$. delemar etc. lead in causing life-threatening illness among them, followed by Mucor and Lichtheimia. These fungi spread from initial site and can cause systemic infection, which affect- lungs, kidney, bone, bladder, gastrointestinal tract, skin, heart, brain etc. Based on the site of infection, invasive mucormycosis is categorised into following types, including- (1) Rhinocerebral/ sino-orbital (in sinus and brain; also called as rhinocerebral mucormycosis; RCM), (2) Pulmonary (lung), (3) Gastrointestinal, (4) Cutaneous (skin) and (5) Scattered/disseminated, occurs when infection spread to other parts of the body through bloodstream
[9]. In COVID-19 patients, the rhino-orbital mucormycosis is chiefly prevalent, followed by orbito-cerebral and the mucormycosis in lungs is seen in $10 \%$ of cases only. Among these, rhino-orbital infections are the deadliest and result in more than $50 \%$ mortality. Thus, timely identification of mucormycosis becomes important. Diagnosis relies on symptoms, culture, and histopathology from sinus sample or debridemented region and computed tomography (CT) $[12$, 13]. PCR of fungal DNA in serum is quicker, than culture or histopathology. Magnetic resonance imaging (MRI) of orbital region, paranasal sinuses and brain, with or without $\mathrm{CT}$ is usually enacted to review magnitude of the infection $[3,13,14]$.

The life cycle of this fungus involves various stages and discussed next, which would help in understanding the spread of infection as well.

\section{Life cycle and reproduction}

Mucorales chiefly grow as hyphae, which are coenocytic, and septa formation is rare. These are haploid (n) fungi, which divide by both asexual and sexual mode of reproduction, as shown in Fig. 1. Asexual reproduction involves sporangia, bearing multiple spores, is the faster mode. These sporangia rupture to release sporangiospores, which forms germ tube and give rise to hyphae like structure (Fig. 1A).
Fig. 1 Life cycle of Zygomycetes fungi. Zygomycetes life cycle have both asexual and sexual phases in their life cycles. A In asexual phase, hyphae produce sporangia bearing numerous haploid sporangiospores, because of mitosis. These sporangia rupture and release spores, which germinate again to form hyphae. $\mathbf{B}$ In the sexual phase, plus and minus haploid (n) mating types conjugate and create a heterokaryotic zygosporangium (2n), which fuse to form a diploid zygote formation, as a result of karyogamy

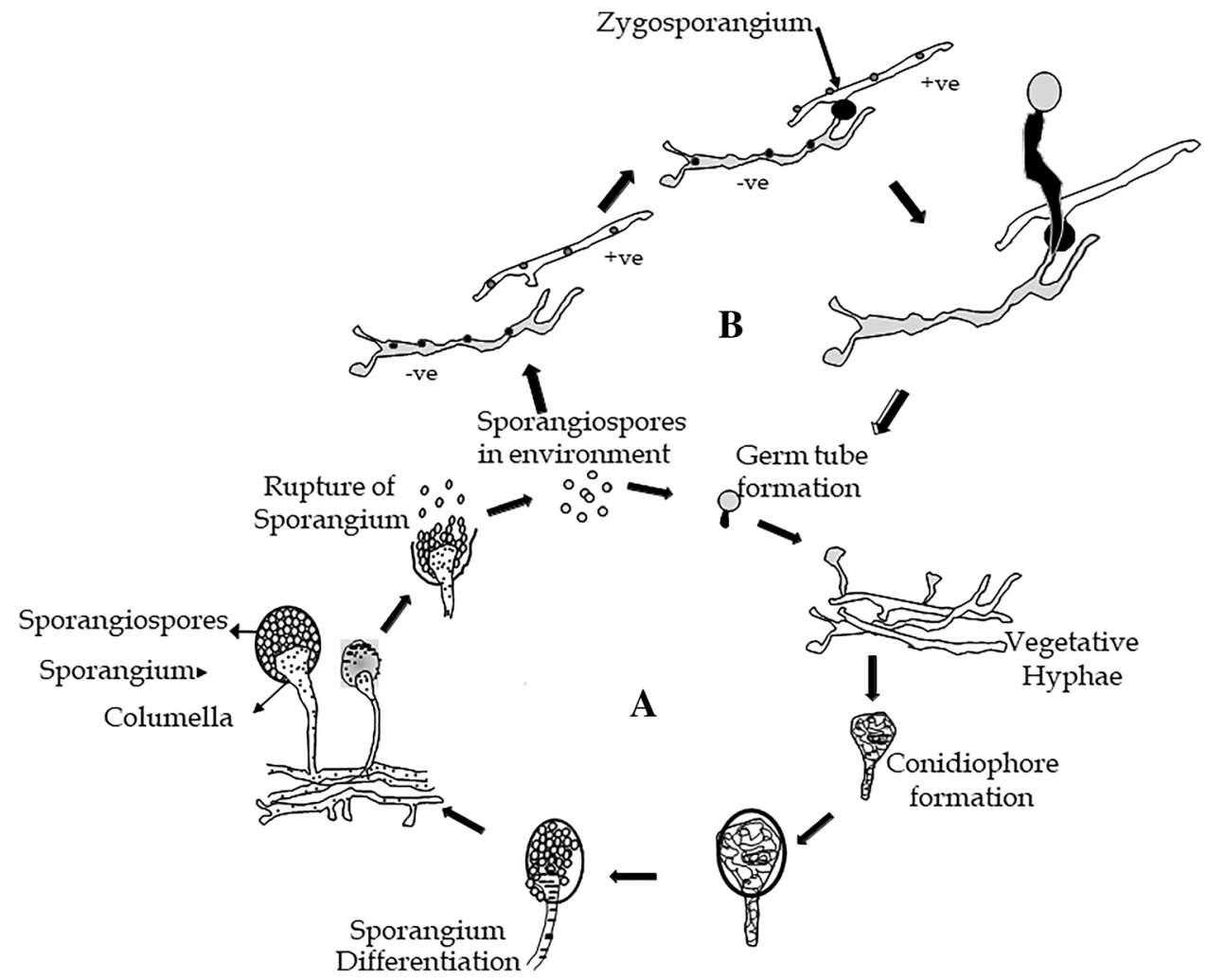


Variation in structure of columella is common among different species [15]. The sexual reproduction involves fusion of opposed sexual spores (zygospores), called gametangia; followed by zygotic meiosis (Fig. 1B). These gametangia arise either from a single mycelium (homothallic); or from separate thalli (heterothallic). Sexually compatibility is determined from expression of a transcription factor (TF) at the mating locus, having two alleles: $\operatorname{sexP}$ for the $(+)$ and sexM for the (-) mating types respectively. Dissimilar TF expressing cells fuse to form a black and thick-walled zygospore, which is able to survive adverse conditions (Fig. 1B). On return of favourable conditions, the zygote spore germinates to form hyphae, which produces sporangium after meiosis. In spite of both modes of reproduction, the asexual sporangiospores are supposed to be the source of infection/ dissemination and might be the preferred mode inside humans, due to its faster rate of multiplication, and discussed next.

\section{Molecular mechanism of mucormycosis in COVID-19 patients}

The time gap between diagnosing COVID-19 infection and contracting mucor infections is around 15 days $[1,12,16]$. The entry of fungi usually begins with inhalation of sporangiospores from air/dust through nose (sometimes from ears) (Fig. 2), although consumption of contaminated food, entry through cuts/ abrasion in the skin, infection from medical devices (linen, bandages) and ventilation system can also lead to contracting infection. These fungi although harmless, become life threatening in immune-compromised patients, having underlying conditions like transplant, corticosteroids therapy, hematologic malignancies, hyperglycaemia, ketoacidosis etc. Studies on the Rhizopus infections implies that, for the rationales still unidentified, spores inhalation in hematologic malignancies (and neutropenic) patients cause pulmonary infections, whereas patients with diabetic ketoacidosis (DKA) are more prone to RCM [17].

Findings on $R$. delemar causing RCM in DKA animal models show that, molecular recognition between fungal CotH3 (Homolog of bacterial spore coat protein) protein and mammalian nasal Glucose-regulated protein 78 (GRP78), promotes adherence in the first stage of infection (Fig. 2). Here, the CotH surface proteins might be functioning as invasions enzymes to promote infection [15]. Its importance is determinable from the fact that anti-CotH3 antibodies provide protection in DKA murine model for mucormycetes infection. Additionally, R. oryzae CotH3 protein expression on the non-pathogenic yeast $S$. cerevisiae, lead to mammalian cell's invasion [18].

In the successive step of infection, patients with high glucose, iron, and ketone body levels provide a suitable microenvironment for heightened expression of both CotH3 and GRP78, leading to trapping of fungi inside sinus cavities (Fig. 2) [10, 18]. Here, fungal cells elongate into tube-like hyphal strands, spreading into the sinuses, lungs, skin, soft tissues and blood stream, causing tissue and epithelium penetration. In the advanced stage, angio-invasion (entry into blood vessels) (Fig. 2) lead to spread of infection to jaws, bones, nerve and brain, which

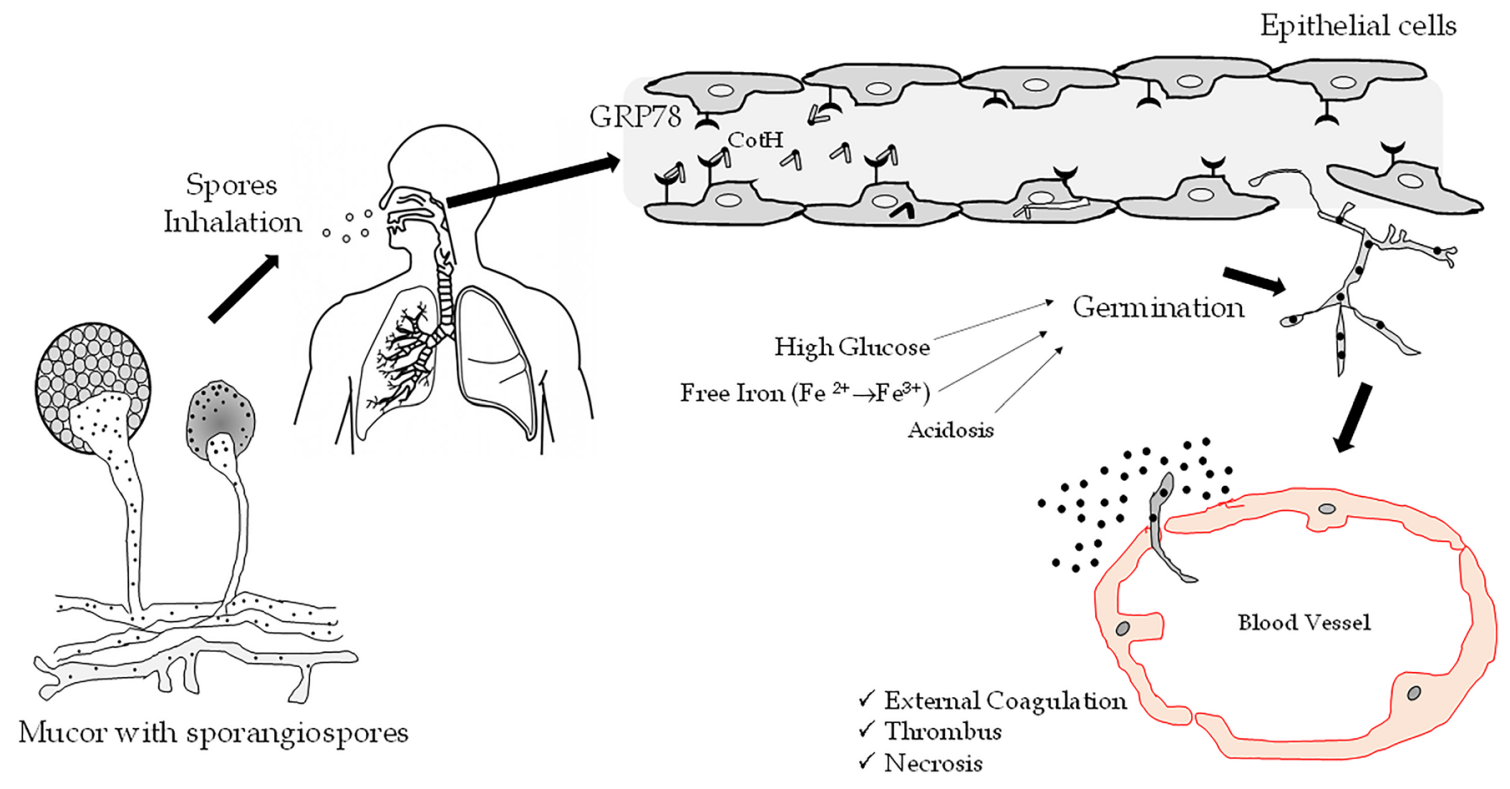

Fig. 2 In rhino-cerebral mucormycosis (RCM), fungal cells spore coat protein CotH3 recognises the mammalian Glucose-regulated protein 78 (GRP78) on nasal epithelial cells to invade, colonize and lyse host cells. The infection spreads to other sites causing inflammation, thrombosis and angioinvasion 
cause blurred vision and ocular alterations $[9,10]$. It is understood that during this process, fungal cells require iron, which is sequestered with iron binding proteins in healthy people, whereas acidosis results in dissociation of iron from sequestering protein and release of free iron $\left(\mathrm{Fe}^{3+}\right)$ [19]. All these factors, as shown in Fig. 2, support the fungal growth and lysis of blood vessels, which results in thrombosis and necrosis of the affected tissue. Notably, presence of CotH fungal protein is limited to Mucorales surface only, and could be the reason behind its better angio-invasive properties than other fungi [17]. In pulmonary mucormycosis, another fungal protein CotH7 helps in gaining entry to lungs, by interacting with host' laminin and collagen IV of basement membrane [17]. Apart from CotH, other components like cell wall, spore germination proteins, iron-sequestration enzymes and host immune evasion in phagocytes also are responsible for pathology of this fungus [10]. Polymorphonuclear neutrophils (PMNs) provide the first line of defence after infection. In subsequent step, PMNs secrete interleukin-1 beta (IL-1 $\beta$ ), induce production of pro-inflammatory cytokines, mediate Th17 differentiation, along with secretion of IL-10, TNF- $\alpha$, IL-6, IL-12, activation of natural killer (NK) cells, acute phase proteins production, antibody secretion and dendritic cells (DC) maturation. The phagocytic cells in DKA condition are not able to contain the infection, its spread leads to establishment of disease and finally once it reaches to brain, leads to mortality in patients. Apart from the normal course of infection, the main risk factors which resulted in an epidemic like situation in some populations is imperative to be highlighted and is taken up in next section.

\section{Risk factors of mucormycetes infections, particularly in COVID-19 patients}

Until now, mucormycetes infection were generally related with renal failure, haematological disorders and intensive care unit (ICU) admission [9]. However, the unforeseen recent rise of mucormycosis in COVID-19 patients led to exploration of the differences in physiology of normal and COVID-19 patients. This culminates in delineation of multiple factors, studied earlier in isolated studies and now compiled in Fig. 3. Initially, it appeared that, lungs being the primary target in COVID-19 disease, thus patients requiring oxygen supply are provided such through pipelines, along with humidifiers and a possible seepage (iatrogenic) with any localized fungal growth could be the triggering factor. This is possible as smaller hospitals use a central oxygen system, and several oxygen points originate from this common system, under emergency conditions. On top of this, COVID-19 patients already have a weakened immune system and disease exhaustion leaves them vulnerable to contracting even rare infections, which develop and spread easily, with full-fledged symptoms. The spread of infection can also occur through contaminated medical devices and linens (nosocomial infections). The patients with underlying liver and kidney ailments are also susceptible to mucorales infection. The cancer and organ transplant patients are also at the edge in developing infection. Besides these, many other factors are crucial key regulatory elements and discussed separately below.

\section{Diabetes mellitus and ketoacidosis}

The underlying uninhibited diabetes mellitus (DM) in COVID-19 patients, results in high sugar levels. This
Fig. 3 List of the various factors contributing to higher incidence of mucormycosis in COVID-19 patients

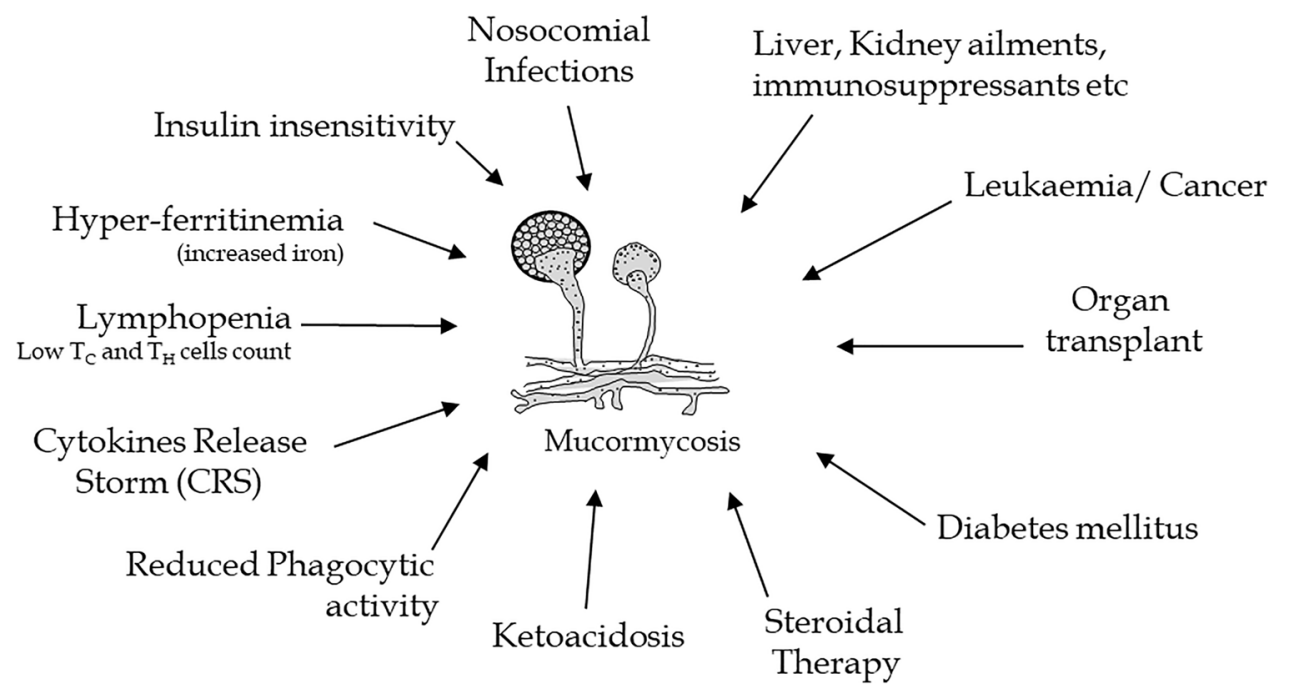


uncontrolled glycaemic level impedes the viral clearance and reduces T-cell function (both Cytotoxic $\mathrm{T}$ cells $(\mathrm{Tc}$ ) and Helper T cells (Th) cells), which lowers immune response $[13,20]$. On top of that, SARS-CoV-2 virus infection management includes rampant steroids administration, which although is life-saving in shrinking the lungs inflammation and controlling damage due to an overdriven and deregulated body's immune system in COVID-19 patients [11]. But steroidal therapy is long known to cause hyperglycaemia in people, and thus combination of existing diabetes DM and steroidal therapy in COVID-19 patients, results in extremely high sugar levels, reaching more than $450-500 \mathrm{mg} / \mathrm{dL}$ [21, 22]. The body cannot utilize this high blood sugar due to limited insulin release, leading to alternative fat metabolism and resulting in ketoacidosis. Both high sugar and acidic blood, generates a luxuriant atmosphere for mucorales fungi to flourish [23]. Reports suggest that mere 2-3 weeks of steroidal therapy and prolonged ICU stays are enough to lower the immunity substantially and increases susceptibility of patients to mucor infections. The ketoacidosis and ketonemia in COVID-19 patients, also weakens phagocytic activity and patients with scarce phagocytes or with reduced phagocytic activity are at greater risk of mucormycosis [24].

\section{Cytokine release storm (CRS) and lymphopenia}

Severe COVID-19 disease patients typically display a delayed innate immune response in recognition of virus, which culminates in a deferred but heightened response against SARS-CoV-2. This allows the virus to multiply exponentially and, in a comeback, retort the host releases interferon's (IFNs). These IFNs activate many cells likedendritic cells (DC), macrophages and in turn secrete various cytokines like IL-6 (Interleukin-6), IL-1, IL-8, IL-12, TNF- $\alpha$, IL-17 etc., termed as cytokine release storm (CRS) and results in lymphopenia due to various mechanisms, including exhaustion phenotype and programmed cell death in lymphocytes [11]. Thus, results in decrease in population of both Th and Tc cells, which are chief warriors of acquired immune system. These cells are important in clearing the fungal cells as well, thus it is not surprising that a general weakened immune system is not enough and fast growing mucor fungi spreads very rapidly in body.

\section{Dysregulated iron metabolism}

IL-6, IL-1 and TNF- $\alpha$ released in CRS are pro-inflammatory cytokines and results in inflammation. The elevated levels of IL-6 almost became a parameter in ascertaining the severity of COVID-19 infection [11]. COVID-19 and IL-6 led hyperinflammation also known to results in iron-dysregulation, which manifest as hyper-ferritinemia. Ferritin is the main site of iron storage and stores iron as $\mathrm{Fe}^{3+}[25]$. The normal ferritin levels are around $250 \mathrm{ng} / \mathrm{ml}$ and in critical patients of COVID-19, including non-survivors, the levels are more than twofold (450-1140 ng/ml) [26]. The IL-6 increases ferritin and hepcidin synthesis in humans. Hepcidin, an iron regulatory hormone is released from liver and limit iron to certain cells, including- macrophages and enterocytes by decreasing cellular iron export via FPN1 (iron exporter ferroportin 1) transporter [25]. This results in high intracellular levels of iron, which interacts with cellular reactive oxygen species (ROS), reactive nitrogen species (RNS) and reactive sulphur species (RSS) in iron dependent peroxidation mechanisms. Ultimately, the increased intracellular iron leads to ferroptosis (programmed cell death) and tissue injury. This led to ferritin discharge into the circulation and here ferritin liberates its iron, producing extremely high levels of free iron in blood. Thus, iron-chelators, like deferoxamine is administered to COVID-19 patients for regulating iron levels [27]. Although chelators effectively reduce serum iron levels, but improperly supplies iron to mucormycetes, which acts as a fuel to their growth $[28,29]$. These conditions supply iron to otherwise iron-starved mucormycetes fungi. These events support mucorales infection, which progresses rapidly ( $4-5 \mathrm{~cm}$ in a day) and soon reaches the brain (rhino-orbito-cerebral).

\section{Insulin resistance}

Interestingly, prolonged IL-6 exposure leads to decreased glucose sensitivity and inhibition of insulin signalling. This is due to the suppression of alpha serine/threonine-protein kinase (AKT) signalling and explained in Fig. 4. Actually, elevated levels of IL-6 bind to its receptor IL-6R, leading to JAK-STAT pathway, involving STAT3 dimerization and enhanced levels of suppressor of cytokine signalling 3 (SOCS-3), which tags the Insulin Receptor Substrate 1 (IRS-1) protein for ubiquitin mediated proteasomal degradation. This finally results in failure of transport of Glucose Transporter type 4 (GLUT-4) carrying vesicles to plasma membrane of cells. Thus, cells fail to uptake glucose inside, leading to higher glucose in blood, resulting in Type 2 Diabetes Mellitus (T2DM) (Fig. 4) [30, 31]. Besides that, in COVID-19 patients, $\beta$-islet cells of pancreas possibly are infected and might result in reduced insulin discharge. In the attempt to bring the high sugar under control, dose of insulin, reaches to $20-40 \mathrm{U} / \mathrm{h}$ and is oddly excessive, as an average diabetic person is dose never reaches more than 80 U/day.

All these factors culminate and create a dangerous combination, which support mucormycetes growth and its related mortality. The physiological parameters in recovered COVID-19 patients also take time to return to normal and thus still exist as a susceptible group. Ultimately, this quick 
Fig. 4 Pathway involving IL-6 and insulin resistance. Elevated levels of IL-6 bind to its receptor IL-6R, leading to JAK-STAT pathway involving STAT3 dimerization and enhanced levels of SOCS-3, which tag the IRS-1 protein for ubiquitinbased degradation. This finally results in failure of transport of GLUT-4 carrying vesicles to plasma membrane of cells and suspension of uptake of glucose into cells, leading to high glucose levels in blood

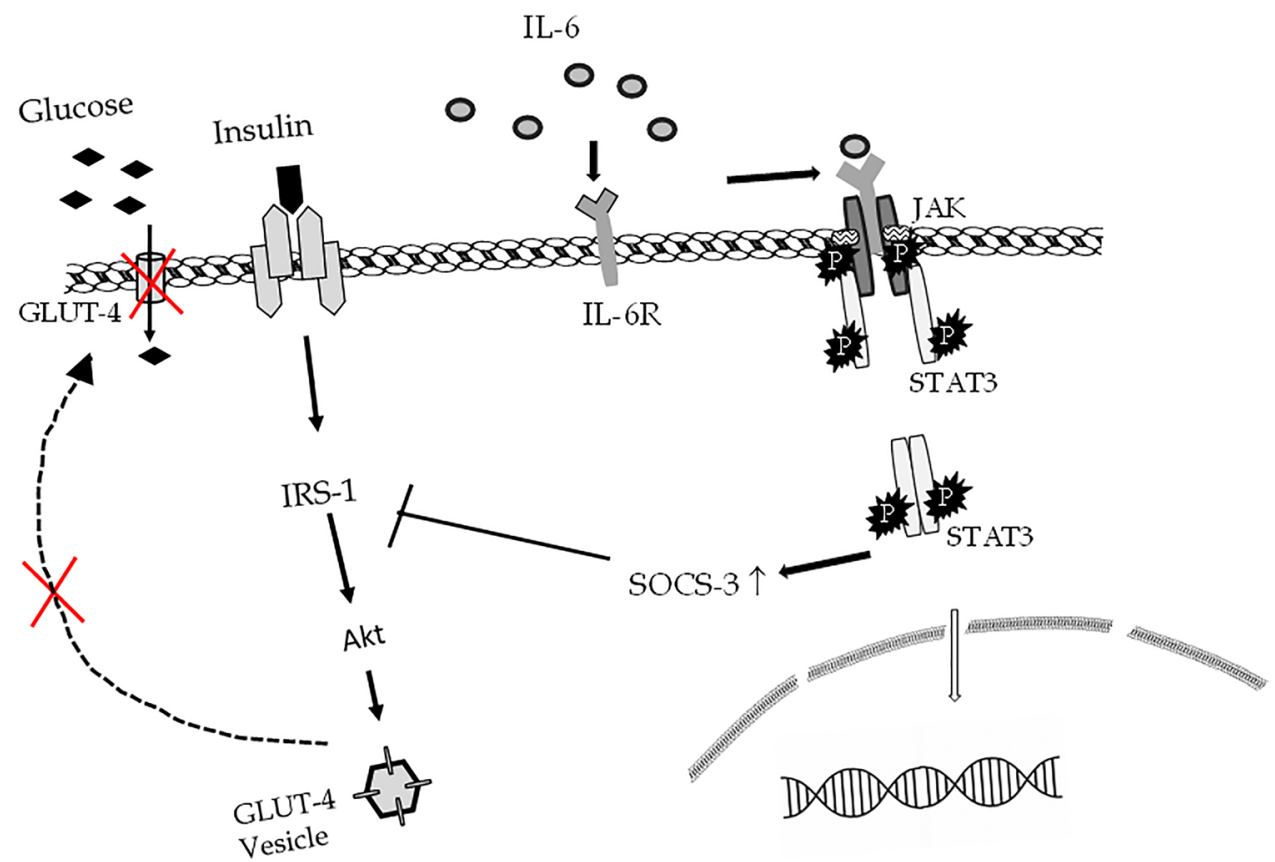

spread of fungus warrants a hard-hitting treatment with antifungals and surgical removal. The treatment options for mucormycosis are also limited and discussed below.

\section{Remedial measures, treatment and conclusions}

The life-threatening RCM in COVID-19 patients generally starts with throbbing headache, fever, facial and nasal pain or numbness, redness around the eyes and/or nose and blackish nasal discharge. This soon follows toothache, loosening of teeth, jaws swelling, eyesight loss and occasionally face paralysis etc. In extreme cases, when the infection spreads further, then removal of the eyes or the partial/full jaw bones becomes the only life-saving option. Multiple sites getting affected demands the support of ENT, neurology, dental and ophthalmology departments at once and makes the situation severe. On top, mucormycosis has a poor prognosis, so prevention is definitely better than cure. Thus, COVID19 recovered patient's needs to be instructed for staying indoors and re-establish their resistance, as well as reduce their fungal exposure from environment. The hot and humid conditions, oxygen masks promote the spread of mucormycetes, so, another prophylactic measure is to advise healthcare workers and technical staff in ICU wards for frequent change of flow meters and periodically sanitization of oxygen tubing. Additionally, it is imperative that COVID-19 patients, kept on high doses of steroids and antibiotics are regularly monitored for hyperglycaemia and acidosis. Even
COVID-19 recovered patients should have follow-up of their sugar levels for a few months.

The major antifungals like azoles and echinocandins are not effective against zygomycetes. Thus, timely diagnosed patients infected with mucorales are often injected with liposomal amphotericin B injections ( $5 \mathrm{mg} / \mathrm{kg} /$ day) for at least 10 days to several weeks $[9,12,16]$. However, it is important to mention that amphotericin B has substantial side effects, especially kidney damage (nephrotoxicity). Along with that, its swift administration along with steroid therapy can also lead to hypokalaemia, a condition of low levels potassium $\left(\mathrm{K}^{+}\right)$in the blood serum and may lead to cardiac dysfunction [32-34]. Thus, it is evident that a multi-professional squad including ophthalmologist, neurosurgeon, critical care specialist, microbiologist, cardiologists and pathologist is fundamental in successful management of mucormycosis. In an overburdened and exhausted health care structure, locating hospitals where mucormycosis requires further surgical and follow-up care is another trauma. Thus, there is a need to identify new targets and drugs for management of rare infections. These unpredicted infections are creating extra distress for physically, emotionally, and financially drained patients from recently transpired COVID-19 infection too.

Author contributions RP conceptualized, performed the literature search and wrote the manuscript. MN also performed literature search, supervised the writing and style correction of the manuscript. Both authors have read and approved the final manuscript.

Funding R. Pasrija acknowledges Science \& Engineering Research Board (SERB) (CRG/2020/004986) and Maharshi Dayanand 
University, Radhakrishnan (R.K.Fund) minor research project (Sanctioned amount: Rs. 50,000) for funding.

Data availability None to declare.

\section{Declarations}

Conflict of interest The authors declare that there are no conflicts of interest.

Ethical approval Not needed in current study.

\section{References}

1. Pal R, Singh B, Bhadada SK et al (2021) COVID-19-associated mucormycosis: an updated systematic review of literature. Mycoses 64:1452-1459. https://doi.org/10.1111/myc.13338

2. Gupta A, Sharma A, Chakrabarti A (2021) The emergence of post-COVID-19 mucormycosis in India: can we prevent it? Indian J Ophthalmol 69:1645-1647. https://doi.org/10.4103/ ijo.IJO_1392_21

3. Millon L, Scherer E, Rocchi S, Bellanger A-P (2019) Molecular strategies to diagnose mucormycosis. J Fungi 5:24. https://doi. org/10.3390/jof5010024

4. Divakar PK (2021) Fungal taxa responsible for mucormycosis/"Black Fungus" among COVID-19 patients in India. J Fungi (Basel) 7:641. https://doi.org/10.3390/jof7080641

5. Chakrabarti A, Singh R (2014) Mucormycosis in India: unique features. Mycoses 57:85-90. https://doi.org/10.1111/myc.12243

6. Prakash H, Skiada A, Paul RA et al (2021) Connecting the dots: interplay of pathogenic mechanisms between COVID-19 disease and mucormycosis. J Fungi 7:616. https://doi.org/10.3390/jof70 80616

7. Mahalaxmi I, Jayaramayya K, Venkatesan D et al (2021) Mucormycosis: an opportunistic pathogen during COVID-19. Environ Res 201:111643. https://doi.org/10.1016/j.envres.2021.111643

8. Nagesh CP (2021) The "black fungus" through a gray lens: imaging COVID-19-associated mucormycosis. Indian J Ophthalmol 69:1648-1649. https://doi.org/10.4103/ijo.IJO_1506_ 21

9. Petrikkos G, Skiada A, Lortholary O et al (2012) Epidemiology and clinical manifestations of mucormycosis. Clin Infect Dis 54(Suppl 1):S23-34. https://doi.org/10.1093/cid/cir866

10. Morales-Franco B, Nava-Villalba M, Medina-Guerrero EO et al (2021) Host-pathogen molecular factors contribute to the pathogenesis of Rhizopus spp. in diabetes mellitus. Curr Trop Med Rep 8:6-17. https://doi.org/10.1007/s40475-020-00222-1

11. Pasrija R, Naime M (2021) The deregulated immune reaction and cytokines release storm (CRS) in COVID-19 disease. Int Immunopharmacol 90:107225. https://doi.org/10.1016/j.intimp. 2020.107225

12. Garg D, Muthu V, Sehgal IS et al (2021) Coronavirus Disease (Covid-19) Associated Mucormycosis (CAM): case report and systematic review of literature. Mycopathologia 186:289-298. https://doi.org/10.1007/s11046-021-00528-2

13. Shakir M, Maan MHA, Waheed S (2021) Mucormycosis in a patient with COVID-19 with uncontrolled diabetes. BMJ Case Rep 14:e245343. https://doi.org/10.1136/bcr-2021-245343

14. Mohammadi F, Badri M, Safari S, Hemmat N (2021) A case report of rhino-facial mucormycosis in a non-diabetic patient with COVID-19: a systematic review of literature and current update. BMC Infect Dis 21:906. https://doi.org/10.1186/ s12879-021-06625-3

15. Morin-Sardin S, Nodet P, Coton E, Jany J-L (2017) Mucor: a Janus-faced fungal genus with human health impact and industrial applications. Fungal Biol Rev 31:12-32. https://doi.org/10. 1016/j.fbr.2016.11.002

16. Sen M, Lahane S, Lahane TP et al (2021) Mucor in a viral land: a tale of two pathogens. Indian J Ophthalmol 69:244-252. https://doi.org/10.4103/ijo.IJO_3774_20

17. Alqarihi A, Gebremariam T, Gu Y et al (2020) GRP78 and integrins play different roles in host cell invasion during mucormycosis. MBio 11:e01087. https://doi.org/10.1128/mBio.01087-20

18. Alspaugh JA (2014) Hostile takeover: fungal protein promotes host cell invasion. J Clin Invest 124:74-76. https://doi.org/10. 1172/JCI73585

19. Swain SK, Lenka S, Das SR (2020) Rhino-orbital mucormycosis—a dreaded clinical entity. IJCRR 12:197-203. https://doi. org/10.31782/IJCRR.2020.122434

20. Vellingiri B, Jayaramayya K, Iyer M et al (2020) COVID19: a promising cure for the global panic. Sci Total Environ 725:138277. https://doi.org/10.1016/j.scitotenv.2020.138277

21. Tamez-Pérez HE, Quintanilla-Flores DL, Rodríguez-Gutiérrez $\mathrm{R}$ et al (2015) Steroid hyperglycemia: prevalence, early detection and therapeutic recommendations: a narrative review. World J Diabetes 6:1073-1081. https://doi.org/10.4239/wjd. v6.i8.1073

22. Gianchandani R, Esfandiari NH, Ang L et al (2020) Managing hyperglycemia in the COVID-19 inflammatory storm. Diabetes 69:2048-2053. https://doi.org/10.2337/dbi20-0022

23. Baldin C, Ibrahim AS (2017) Molecular mechanisms of mucormycosis-the bitter and the sweet. PLoS Pathog 13:e1006408. https://doi.org/10.1371/journal.ppat.1006408

24. Ibrahim AS, Spellberg B, Walsh TJ, Kontoyiannis DP (2012) Pathogenesis of mucormycosis. Clin Infect Dis 54:S16-22. https://doi.org/10.1093/cid/cir865

25. Edeas M, Saleh J, Peyssonnaux C (2020) Iron: innocent bystander or vicious culprit in COVID-19 pathogenesis? Int J Infect Dis 97:303-305. https://doi.org/10.1016/j.ijid.2020.05. 110

26. Onur ST, Altın S, Sokucu SN et al (2021) Could ferritin level be an indicator of COVID-19 disease mortality? J Med Virol 93:1672-1677. https://doi.org/10.1002/jmv.26543

27. Sonnweber T, Boehm A, Sahanic S et al (2020) Persisting alterations of iron homeostasis in COVID-19 are associated with non-resolving lung pathologies and poor patients' performance: a prospective observational cohort study. Respir Res 21:276. https://doi.org/10.1186/s12931-020-01546-2

28. Spellberg B, Edwards J, Ibrahim A (2005) Novel perspectives on mucormycosis: pathophysiology, presentation, and management. Clin Microbiol Rev 18:556-569. https://doi.org/10.1128/ CMR.18.3.556-569.2005

29. Ibrahim AS, Spellberg B, Edwards J (2008) Iron acquisition: a novel perspective on mucormycosis pathogenesis and treatment. Curr Opin Infect Dis 21:620-625. https://doi.org/10.1097/QCO. 0b013e3283165fd1

30. Kim J-H, Bachmann RA, Chen J (2009) Interleukin-6 and insulin resistance. Vitam Horm 80:613-633. https://doi.org/ 10.1016/S0083-6729(08)00621-3

31. Sindhughosa D, Pranamartha A (2017) The involvement of proinflammatory cytokines in diabetic nephropathy: focus on interleukin 1 (IL-1), interleukin 6 (IL-6), and tumor necrosis factor-alpha (TNF- $\alpha$ ) signaling mechanism. Bali Med J 6:44-51. https://doi.org/10.15562/bmj.v6i1.299

32. Randhawa G, Hagaman S, Pourabdollah Tootkaboni M et al (2021) A rare case of invasive mucormycosis in a diabetic 
patient treated with a short course of dexamethasone. Am J Case Rep 22:932129. https://doi.org/10.12659/AJCR.932129

33. Takazono T, Tashiro M, Ota Y et al (2020) Factor analysis of acute kidney injury in patients administered liposomal amphotericin B in a real-world clinical setting in Japan. Sci Rep 10:15033. https://doi.org/10.1038/s41598-020-72135-y

34. Shamanna K, Fathima A, Sowjanya S (2019) Rhino-orbitocerebral mucormycosis: our experience. Res Otolaryngol 8:2529. https://doi.org/10.5923/j.otolaryn.20190802.03
Publisher's Note Springer Nature remains neutral with regard to jurisdictional claims in published maps and institutional affiliations. 